\title{
Genetic Algorithm Calibration for Two Objective Scheduling Parallel Jobs on Hierarchical Grids
}

\author{
Victor Hugo Yaurima-Basaldua ${ }^{1}$, Andrei Tchernykh ${ }^{2}$, Yair Castro-Garcia ${ }^{3}$, \\ Victor Manuel Villagomez-Ramos ${ }^{1}$, and Larisa Burtseva ${ }^{3}$ \\ 1 CESUES Superior Studies Center, San Luis R.C., Mexico \\ vyaurima@yahoo.com, vicmvr@msn.com \\ ${ }^{2}$ CICESE Research Center, Ensenada, Mexico \\ chernykh@cicese.mx \\ 3 Autonomous University of Baja California, Mexicali, Mexico \\ yair.castro.garcia@gmail.com, lpb@iing.mxl.uabc.mx
}

\begin{abstract}
This paper addresses non-preemptive offline scheduling parallel jobs on a Grid. We consider a Grid scheduling model with two stages. At the first stage, jobs are allocated to a suitable Grid site, while at the second stage, local scheduling is independently applied to each site. In this environment, one of the big challenges is to provide a job allocation that allows more efficient use of resources and user satisfaction. In general, the criteria that help achieve these goals are often in conflict. To solve this problem, two-objective genetic algorithm is proposed. We conduct comparative analysis of five crossover and three mutation operators, and determine most influential parameters and operators. To this end multi factorial analysis of variance is applied.
\end{abstract}

Keywords: Offline scheduling, Grid, Genetic Algorithm, Crossover Operator, Mutation Operator.

\section{Introduction}

In this paper, we present experimental work in the field of multi-objective scheduling in a two layer Grid computing. At the first layer, we select the best suitable machine for each job using a given criterion. At the second layer, local scheduling algorithm is applied to each machine independently. In such an environment, one of the big challenges is to provide scheduling that allows more efficient use of resources, and satisfies other demands. The optimization criteria are often in conflict. For instance, resource providers and users have different objectives: providers strive for high utilization of resources, while users are interested in a fast response. We provide solutions that consider both goals. The aggregation method of criteria and a scalar function to normalize them are used. We examine the overall Grid performance based on real data and present a comprehensive comparative analysis of five crossover operators, three operators of 
mutation, five values of crossover probability, and five values of mutation probability. To genetic algorithm tune up the multifactorial analysis of variance is applied.

After formally presenting our Grid scheduling model in Section 2, we discuss related work in Section 3. We introduce genetic scheduling algorithms and discuss their application for Grid scheduling in Section 4. Genetic algorithm calibration is presented in section 5. Finally, we conclude with the summary in Section 6.

\section{Model}

We address an offline scheduling problem: $n$ parallel jobs $J_{1}, J_{2}, \ldots, J_{n}$ must be scheduled on $m$ parallel machines (sites) $N_{1}, N_{2}, \ldots, N_{m}$. Let $m_{i}$ be the number of identical processors or cores of machine $N_{i}$. Assume without loss of generality that machines are arranged in non-descending order of their numbers of processors, that is $m_{1} \leq m_{2} \leq \ldots \leq m_{m}$ holds.

Each job $J_{i}$ is described by a tuple $\left(\right.$ size $\left._{j}, p_{j}, p_{j}^{\prime}\right)$ : its size $1 \leq$ size $_{j} \leq m_{m}$ also called processor requirement or degree of parallelism, execution time $p_{j}$ and user runtime estimate $p_{j}^{\prime}$. The release date of a job is zero; all the jobs are available before scheduling process start. Job processing time is unknown until the job has completed its execution (non-clairvoyant case). User runtime estimate $p_{j}^{\prime}$ is provided by a user. A machine must execute a job by exclusively allocating exactly size $_{j}$ processors for an uninterrupted period of time $p_{j}$ to it. As we do not allow multisite execution and co-allocation of processors from different machines, a job $J_{j}$ can only run on machine $N_{i}$ if size $\leq m_{j}$ holds.

Two criteria are considered: makespan: $C_{\max }, C_{\max }=\max \left(C_{i}\right)$, where $C_{i}$ is the maximum completion time on $N_{i}$ machine $\left(i=1,2,3, \ldots, N_{m}\right)$ and mean turnaround time: $T A=\frac{1}{n} \sum_{j=1}^{n} c_{j}$, where $c_{j}$ is the completion time of job $J_{j}$.

We denote our Grid model by $G P_{m}$. In the three field notation $(\alpha|\beta| \gamma)$ introduced in [6], our scheduling problem is characterized as $G P_{m}\left|s i z e_{j}, p_{j}, p_{j}^{\prime}\right| O W A$, where $O W A$ is the value of the multi-criteria aggregation operator $(O W A=$ $\left.w_{1} C_{\max }+w_{2} T A\right)$, and $w_{i}$ is the linear combination weights. The problem of scheduling on the second stage is denoted as $P_{m}\left|s i z e_{j}, p_{j}, p_{j}^{\prime}\right| C_{\max }$.

\section{Related Work}

\subsection{Hierarchical Scheduling}

Scheduling algorithms for two layer Grid models can be split into a global allocation part and a local scheduling part. Hence, we regard MPS (Multiple machine Parallel Scheduling) as a two stage scheduling strategy: $M P S=$ $M P S \_$Alloc + PS [20]. At the first stage, we allocate a suitable machine for each job using a genetic algorithm. At the second stage, $P S$ (single machine Parallel Scheduling) algorithm is applied to each machine independently for jobs allocated during the previous stage. 


\section{$3.2 \quad$ Multi-criteria Scheduling}

Several studies deal with scheduling in Grids considering only one criterion (e.g. EGEE Workload Management System [1], NorduGrid broker [4], eNANOS [16], Gridway [10. Various Grid resource managements involve multiple objectives and may use multicriteria decision support. Dutot et al. 3. considered task scheduling on the resources taking into account maximum task completion time and the average completion time. Siddiqui, et al. [18, presented task scheduling based on the resource negotiation, advance reservation, and user preferences. The user preferences are modeled by utility functions, in which users must enter the values and levels of negotiation.

General multi-criteria decision methodology based on the Pareto optimality can be applied. However, it is very difficult to achieve fast solutions needed for Grid resource management by using the Pareto dominance. The problem is very often simplified to a single objective problem or objectives' combining. There are various ways to model preferences, for instance, they can be given explicitly by stakeholders to specify an importance of every criterion or a relative importance between criteria. This can be done by a definition of criteria weights or criteria ranking by their importance.

In order to provide effective guidance in choosing the best strategy, Ramirez et al. [15] performed a joint analysis of several metrics according to methodology proposed in [19. They introduce an approach to multi-criteria analysis assuming equal importance of each metric. The goal is to find a robust and well performing strategy under all test cases, with the expectation that it will also perform well under other conditions, e.g., with different Grid configurations and workloads. Kurowski et al. 11] used aggregation criteria method for modeling the preferences of participants (owners, system managers and users). Authors considered two stage hierarchical grids, taking into account the stakeholders' preferences, assuming unknown processing times of the tasks, and studied the impact of the size of the batch of tasks on the efficiency of schedules. Lorpunmanee et al. 12] presented task allocation strategies to the different sites of a Grid and propose a model for task scheduling considering multiple criteria. They concluded that such scheduling can be performed efficiently using GAs.

In this paper, we consider two-criteria scheduling problem. We propose a genetic algorithm as a strategy for allocating jobs to resources. It uses an aggregation criteria method and the weight generating function representing the relative importance of each criterion. We present an experimental analysis of such a problem and compare obtained results with strategies aimed at optimizing a single criterion. In this paper, the Ordered Weighted Averaging $(O W A)$ [11] is applied: $O W A\left(x_{1}, x_{2}, \ldots, x_{n}\right)=\sum_{c=1}^{k} w_{c} s(x)_{\sigma(c)}$, where $w_{c}$ is the weight, $c=1, \ldots, k, x_{c}$ is a value associated with the satisfaction of the $c$ criterion. Permutation ordering values: $s(x)_{\sigma(1)} \leq s(x)_{\sigma(2)} \leq \ldots s(x)_{\sigma(k)}$ is performed. Weights $\left(w_{c}\right)$ are nonnegative and $\sum_{c=1}^{k} w_{c}=1$. If all the weights are set to the same value, $O W A$ behaves as the arithmetic mean. In this case, high values of some criterion compensate low values of the other ones. In $O W A$ approach, a compromise solution is provided. The highest weight is $w_{1}$ and the subsequent ones are 
decreasing, but never reaching 0 . That means that both the worst criterion and the mean of criteria are taken into account. In this way stakeholders are able to evaluate schedules using multiple criteria. The goal is to find a weighting scheme that provides the best possible mean value for all stakeholders' evaluations and the highest possible value for the worst case evaluation. To achieve this, weight $w_{1}$ must be relatively large, while weight $w_{k}$ should be small, where $k$ denotes the number of criteria. The remaining weights decrease in value from $w_{1}$ to $w_{k}$ according to:

$$
w_{c}= \begin{cases}3 / 2 k, & c=1 \\ (3 k-2 c-1) /[2 n(k-1)], & 1<c \leq k\end{cases}
$$

\section{Genetic Algorithm}

Scheduling algorithms for two layer Grid models can be split into a global allocation part and a local scheduling part. Hence, we regard MPS as a two stage scheduling strategy: $M P S=M P S_{-}$Alloc $+P S$. At the first stage, we use a genetic algorithm $(G A)$ to allocate a suitable machine for each job $\left(M P S_{-} A l l o c=G A\right)$. At the second stage, the parallel job scheduling algorithm $P S$ is applied to each machine independently for jobs allocated during the previous stage. As $P S$ we use the well-known strategy Backfilling-EASY 2122.

$G A$ is a well-known search technique used to find solutions to optimization problems [9]. Candidate solutions are encoded by chromosomes (also called genomes or individuals). The set of initial individuals forms the population. Fitness values are defined over the individuals and measures the quality of the represented solution. The genomes are evolved through the genetic operators generation by generation to find optimal or near-optimal solutions. Three genetic operators are repeatedly applied: selection, crossover, and mutation. The selection picks chromosomes to mate and produce offspring. The crossover combines two selected chromosomes to generate next generation individuals. The mutation reorganizes the structure of genes in a chromosome randomly so that a new combination of genes may appear in the next generation. The individuals are evolved until some stopping criterion is met. $O W A$ operator as a fitness function is applied (Section 2).

Each solution is encoded by $n \cdot m$ matrix. Where $m$ is a number of machines in a Grid, and $n$ is a number of jobs. The $i=0, \ldots, m-1$ row represents local queue of machine $N_{i}$. Note, that machines are arranged in non-descending order of their number of processors $m_{0} \leq m_{2} \leq m_{m-1}$. A job $J_{j}$ can only run on machine $N_{i}$ if $s i z e_{j} \leq m_{i}$ holds. The available set of machines for a job $J_{j}$ is defined to be the machines with indexes $f_{j}, \ldots, m$, where $f_{j}$ is the smallest index $i$ such that $m_{i} \geq$ size $_{j}$.

The selection picks chromosomes to produce offspring. The binary tournament selection known as an effective variant of the parents' selection is considered. Two individuals are drawn randomly from the population, and one with highest fitness value wins the tournament. This process is repeated twice in order to select two parents. 


\subsection{Crossover Operators}

Crossover operator produces new solutions by combining existing ones. It takes parts of solution encodings from two existing solutions (parents) and combines them into single solution (child). The crossover operator is applied under a certain probability $\left(P_{c}\right)$. In this paper, five operators are considered.

One Segment Crossover for Matrix $(O S X M)$. It is based on the crossover operator OSX - One Segment Crossover 7 . In this crossover, two random points $S 1$ and $S 2$ from 0 to vmax (maximum index used) are selected. The child inherits columns from the 0 to $S 1$ position from parent 1. It also inherits columns from $S 1$ to $S 2$ from parent 2, but only elements that have not been copied from parent 1. Finally, child inherits elements from parent 1 that have not yet been copied.

Two Point Crossover for Matrix (TPM). It is based on the crossover operator Two Point Crossover [13]. In this crossover, two random points $S 1$ and $S 2$ from 0 to vmax are selected. Columns from position 0 to $S 1$ and from $S 2$ to vmax are copied from parent 1 . The remaining elements are copied from the parent 2 only if they have not been copied.

Order Based Crossover for Matrix $(O B X M)$. It is based on the crossover operator OBX - Order Based Crossover [5]. A binary mask is used. The mask values equal to one indicate that the corresponding columns are copied from parent 1 to the child. The rest of elements are copied from parent 2, only if they have not been copied. The mask values are generated randomly and uniformly.

Precedence Preservative Crossover for Matrix (PPXM). It is based on the crossover operator PPX - Precedence Preservative Crossover 2]. Random binary mask values equal to one indicates that corresponding columns are copied from parent 1 to the child, and the values equal to zero indicate that columns are copied from parent 2 , this is done by iterating the columns of parents who have not been copied in order from left to right.

Order Segment Crossover for Matrix with Setup $(O S X M S)$. It is based on the crossover operator OSX - Order Segment Crossover [7]. It chooses two points randomly. Columns from position 1 to the first point are copied from parent 1. The elements are ordered by the number of processors required for subsequent insertion into the child in the position according to the number of required processors. Columns from first point to second point are copied from parent 2, only if the elements have not been copied. Finally, the remaining elements are copied from the parent 1 , considering not copied elements.

\subsection{Mutation}

The mutation operator produces small changes in an offspring with probability $P_{m}$. It prevents falling of all solutions into local optimum and extends search space of the algorithm. Three operators Insert, Swap and Switch adapted for two-dimensional encoding are considered. 1)Queue_Insert. Two points are randomly selected. The element of the second point is inserted to the first point, shifting the rest. Note that this mutation preserves most of the order and the 
adjacency information. 2)Queue_Swap. This mutation randomly selects two points and swaps their elements. 3)Queue_Switch. This mutation selects a random column and swaps their elements with the next column.

\section{GA Calibration}

\subsection{Workload}

The accuracy of the evaluation highly relies upon workloads applied. For testing the job execution performance under a dedicated Grid environment, we use Grid workload based on real production traces. Carefully reconstructed traces from real supercomputers provide a very realistic job stream for simulation-based performance evaluation of Grid job scheduling algorithms. Background workload (locally generated jobs) that is an important issue in non-dedicated Grid environment is not addressed.

Four logs from PWA (Parallel Workloads Archive) [14] (Cornell Theory Center, High Performance Computing Center North, Swedish Royal Institute of Technology and Los Alamos National Lab) and one from GWA (Grid Workloads Archive) 8 (Advanced School for Computing and Imaging) have been used: The archives contain real workload traces from different machine installations. They provide information of individual jobs including submission time, and resource requirements.

For creating suitable grid scenarios, we integrate several logs by merging users and their jobs. The premise for the integration of several logs of machines in production use into a Grid log is based on the following. Grid logs contain jobs submitted by users of different sites; a Grid execution context could be composed by these sites. Unification of these sites into a Grid will trigger to merger users and their jobs. It should be mentioned that merging several independent logs to simulate a computational Grid workload does not guarantee representation of the real Grid with the same machines and users. Nevertheless, it is a good starting point to evaluate Grid scheduling strategies based on real logs in the case of the lack of publicly available Grid workloads. Time zone normalization, profiled time intervals normalization, and invalid jobs filtering are considered.

\subsection{Calibration Parameters}

A method of experimental design is adapted from Ruiz and Maroto [17, where the following steps are defined: (a) test all instances produced with possible combinations of parameters; (b) obtain the best solution for each instance; (c) apply the Multifactor Variance Analysis (ANOVA) with 95\% confidence level to find the most influential parameters; (d) set algorithm parameters based on selected parameters values; (e) calculate relative difference of the calibrated algorithm and other adapted algorithms over the best solutions. Table 1 shows parameters that were set for the calibration. Hence, $5 \times 3 \times 5 \times 5=375$ different algorithms alternatives were considered. 30 executions of the workload were realized, in total $375 \times 30=11,250$ experiments. 
Table 1. Calibration parameters

\begin{tabular}{ll}
\hline Instance & Levels \\
\hline Crossover operators & OSXM, TPM, OBXM, PPXM, OSXMS \\
Mutation operators & Queue_Insert, Queue_Swap, Queue_Switch \\
Crossover probability & $0.001,0.01,0.05,0.1,0.2$ \\
Population & 50 individuals \\
Number of jobs in individual & 5275 \\
Selection & Binary tournament \\
Stop criterion & If the fitness value of the best chromosome is not \\
& improved 4 times, the algorithm is stopped. \\
\hline
\end{tabular}

The performance of the proposed algorithm is calculated as the percentage of the relative distance of the obtained solution from the best one (Relative Increment over the Best Solution - RIBS). The RIBS is calculated using the following formula: $R I B S=\left(H_{e} u_{s o l}-B_{e s t} s_{s o l}\right) /$ Best $_{\text {sol }} \cdot 100$, where $\mathrm{Heu}_{\text {sol }}$ is the value of the objective function obtained by considered algorithm, and Best sol $_{\text {l }}$ is the best value obtained during the testing all possible parameter combinations.

\subsection{Analysis of Variance}

To assess the statistical difference among the experimental results, and observe effect of different parameters on the result quality, the ANOVA is applied. The analysis of variance is used to determine factors that have a significant effect, and which are the most important factors. Parameters of the Grid scheduling problem are considered as factors, and their values as levels. We assume that there is no interaction between the factors.

Table 2. Analysis of variance for RIBS (Type III Sums of Squares)

\begin{tabular}{lccccc}
\hline Source & Sum of Squares & Df & Mean Square & F-Ratio & P-Value \\
\hline MAIN EFFECTS & & & & & \\
A:cross & 2111.03 & 4 & 527.758 & 152.00 & 0.0000 \\
B:mut & 47.4724 & 2 & 23.7362 & 6.84 & 0.0012 \\
C:pc & 20.9586 & 4 & 5.23964 & 1.51 & 0.1990 \\
D:pm & 27.5114 & 4 & 6.87785 & 1.98 & 0.0969 \\
RESIDUAL & 1249.99 & 360 & 3.4722 & & \\
TOTAL & 3456.97 & 374 & & & \\
(CORRECTED) & & & & & \\
\hline
\end{tabular}

The F-Ratio is the ratio between mean square of the factor and the mean square of residues. A high F-Ratio means that this factor affects the response variable (see Table 22). The value of $P$-Value shows the statistical significance of the factors. The factors, whose $P$-Value is less than 0.05 , have statistically significant effect on the response variable $(R I B S)$ with $95 \%$ level of confidence. 
Here we see that the factors that significantly affect the response variable are the operators of crossover and mutation. According to the F-Ratio, the most important factor is the crossover operator.

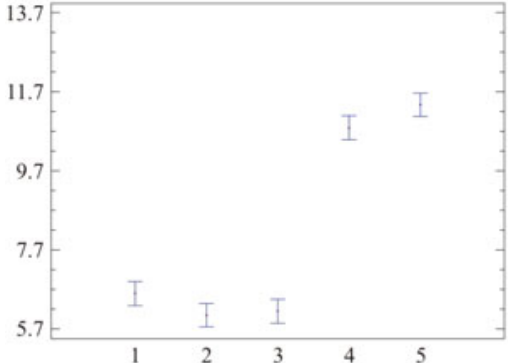

(a) Crossover operators

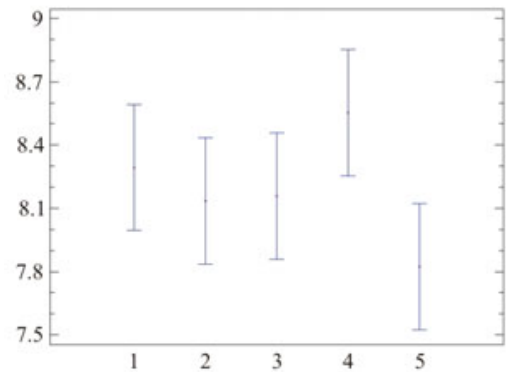

(c) Crossover probability

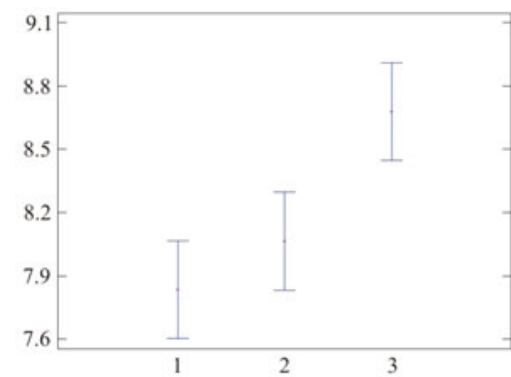

(b) Mutation operators

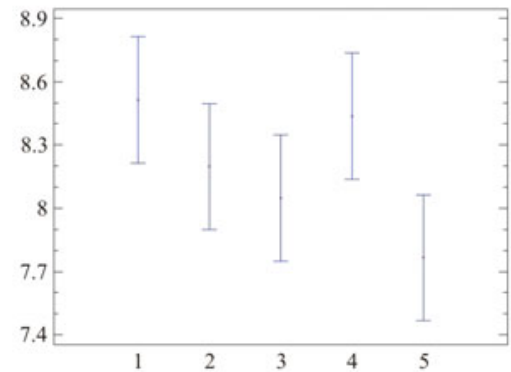

(d) Mutation probability

Fig. 1. Means and 95\% LSD intervals

Figs. 1(a)-1(d) show means and 95\% LSD intervals of the most influential factors. Fig. 1(a) shows the results obtained for crossover operators. Five operators are presented in the following order: 1-OSXM, 2-TPM, 3-OBXM, 4-PPXM and $5-O S X M S$. The vertical axis is the values of $R I B S$. We can see that crossover $T P M$ is the best crossover among the five ones tested, followed by $O B X M$. Figure 1 (b) shows the results obtained for mutation operators. Three mutation operators are presented in the following order: 1- Queue_Insert, 2- Queue_Swap y 3Queue_Switch. Queue_Insert is shown to be the best, followed by Queue_Swap. Figures 1(c) and1(d) show the results for the crossover and mutation probability. The best crossover probability occurring is 0.9 . The best mutation probability occurring is 0.2 .

\section{Conclusions and Future Work}

We addressed a two-objective genetic algorithm calibration for scheduling jobs in a two stage computational Grid. We conduct comprehensive comparison of 
five known crossover operators, three mutation operators adapted for two dimension encoding, five values for the crossover probability, and five values for the mutation probability. 375 different genetic algorithms are analysed with 30 instances for each one. 11,250 experiments were evaluated. We use aggregation criteria method for modeling the preferences of two objectives: $C_{\max }$ and $T A$. To assess the statistical difference among the experimental results, and observe impact of different parameters on the result quality, the ANOVA technique was applied. The crossover operator is shown to have a statistically significant effect. It plays the most important role in genetic algorithms applied to a scheduling in computational Grid. Of five compared operators, the TPM obtained the best result, followed by the $O B X M$.

Obtained results may serve as a starting point for future heuristic Grid scheduling algorithms that can be implemented in real computational Grids. While the scope of this work is to determine most influential GA parameters and operators, in future work, we also intend to evaluate the practical performance of the proposed strategies, and the assessment of their cost. To this end, we plan simulations using real workload traces and corresponding Grid configurations. We will compare our $G A$ with other existing Grid scheduling strategies which are typically based on heuristics. Future work needs for a better understanding of the scheduling with dynamic Grid configuration, resource failures and other real characteristics of the Grid.

\section{References}

1. Avellino, G., Beco, S., Cantalupo, B., Maraschini, A., Pacini, F., Terracina, A., Barale, S., Guarise, A., Werbrouck, A., Di Torino, S., Colling, D., Giacomini, F., Ronchieri, E., Gianelle, A., Peluso, R., Sgaravatto, M., Mezzadri, M., Prelz, F., Salconi, L.: The EU datagrid workload management system: towards the second major release. In: 2003 Conference for Computing in High Energy and Nuclear Physics. University of California, La Jolla (2003)

2. Bierwirth, C., Mattfeld, D., Kopfer, H.: On Permutation Representations for Scheduling Problems. In: Ebeling, W., Rechenberg, I., Voigt, H.-M., Schwefel, H.P. (eds.) PPSN 1996, Part II. LNCS, vol. 1141, pp. 310-318. Springer, Heidelberg (1996)

3. Dutot, P., Eyraud, L., Mounie, G., Trystram, D.: Models for scheduling on large scale platforms: which policy for which application? In: Proceedings of 18th International Symposium on Parallel and Distributed Processing, p. 172 (2004)

4. Elmroth, E., Tordsson, J.: An interoperable, standards based Grid resource broker and job submission service. In: First International Conference on e-Science and Grid Computing, 2005, pp. 212-220. IEEE Computer Society, Melbourne (2005)

5. Gen, M., Cheng, R.: Genetic algorithms and engineering optimization, p. 512. John Wiley and Sons, New York (1997)

6. Graham, R.L., Lawler, E.L., Lenstra, J.K., Rinnooy Kan, A.H.G.: Optimization and approximation in deterministic sequencing and scheduling: a survey. In: Hammer, P.L., Johnson, E.L., Korte, B.H. (eds.) Discrete Optimization II. Annals of Discrete Mathematics, vol. 5, pp. 287-326. North-Holland, Amsterdam (1979) 
7. Guinet, A., Solomon, M.: Scheduling Hybrid Flowshops to Minimize Maximum Tardiness or Maximum Completion Time. Int. J. Production Research 34(6), 16431654 (1996)

8. GWA Grid Workloads Archive, http://gwa.ewi.tudelft.nl

9. Holland, J.: Adaptation in natural and artificial systems. University of Michigan Press (1975)

10. Huedo, E., Montero, R.S., Llorente, I.M.: Evaluating the reliability of computational grids from the end user's point of view. Journal of Systems Architecture 52(12), 727-736 (2006)

11. Kurowski, K., Nabrzyski, J., Oleksiak, A., Wglarz, J.: A multicriteria approach to two-level hierarchy scheduling in Grids. Journal of Scheduling 11(5), 371-379 (2008)

12. Lorpunmanee, S., Noor, M., Hanan, A., Srinoy, S.: Genetic algorithm in Grid scheduling with multiple objectives. In: Proceedings of the 5th WSEAS Int. Conf. on Artificial Intelligence, Knowledge Engineering and Data Bases, Madrid, Spain, pp. 429-435 (2006)

13. Michalewicz, Z.: Genetic Algorithms + Data Structures = Evolutions Programs, 3rd edn. Springer, Heidelberg (1996)

14. PWA Parallel Workloads Archive, http://www.cs.huji.ac.il/labs/parallel/workload/

15. Ramirez-Alcaraz, J.M., Tchernykh, A., Yahyapour, R., Schwiegelshohn, U., Quezada-Pina, A., Gonzalez-Garcia, J.-L., Hirales-Carbajal, A.: Job Allocation Strategies with User Run Time Estimates for Online Scheduling in Hierarchical Grids. J. Grid Computing 9, 95-116 (2011)

16. Rodero, I., Corbalán, J., Badía, R.M., Labarta, J.: eNANOS Grid Resource Broker In: Sloot, P.M.A., Hoekstra, A.G., Priol, T., Reinefeld, A., Bubak, M. (eds.) EGC 2005. LNCS, vol. 3470, pp. 111-121. Springer, Heidelberg (2005)

17. Ruiz, R., Maroto, C.: A genetic algorithm for hybrid flowshops with sequence dependent setup times and machine eligibility. European Journal of Operational Research 169, 781-800 (2006)

18. Siddiqui, M., Villazon, A., Fahringer, T.: Grid Capacity Planning with Negotiationbased Advance Reservation for Optimized QoS. In: Proceedings of the 2006 ACM/IEEE Conference on Supercomputing (SC 2006). ACM, New York, article 103 (2006), http://doi.acm.org/10.1145/1188455.1188563

19. Tsafrir, D., Etsion, Y., Feitelson, D.G.: Backfilling using system-generated predictions rather than user runtime estimates. IEEE Trans. Parallel Distrib. Syst. 18, 789-803 (2007)

20. Tchernykh, A., Schwiegelsohn, U., Yahyapour, R., Kuzjurin, N.: Online Hierarchical Job Scheduling on Grids with Admissible Allocation. Journal of Scheduling 13(5), 545-552 (2010)

21. Lifka, D.A.: The ANL/IBM SP Scheduling System. In: Feitelson, D.G., Rudolph, L. (eds.) IPPS-WS 1995 and JSSPP 1995. LNCS, vol. 949, pp. 295-303. Springer, Heidelberg (1995)

22. Skovira, J., Chan, W., Zhou, H., Lifka, D.: The EASY - LoadLeveler API Project. In: Feitelson, D.G., Rudolph, L. (eds.) IPPS-WS 1996 and JSSPP 1996. LNCS, vol. 1162, pp. 41-47. Springer, Heidelberg (1996) 